\title{
Bryophytes of Mount Abu, Rajasthan, India
}

\author{
Krishna Kumar Rawat*, Vinay Sahu and Ramya Ranjan Paul \\ CSIR-National Botanical Research Institute, Rana Pratap Marg, Lucknow - 226001, India \\ *Corresponding author: drkkrawat@rediffmail.com \\ माउंट आबू, राजस्थान के हरितोद्भिद \\ कृष्ण कुमार रावत, विनय साहू और राम्या रंजन पॉल
}

\section{सारांश}

पूर्व कें किए गए संकलन एवं हाल के अन्वेषण के परिणामों के आधार पर, वर्तमान कार्य माउंट आबू, राजस्थान से संकलित 113 हरितोद्भिद टैक्सा की सूची प्रदान की गई है, जिसमें से 24 टैक्सा माउंट आबू क्षेत्र के लिए नए हैं। इनकें से 14 टैक्सा राजस्थान राज्य के लिए नए अभिलेख हैं, जबकि 07 टैक्सा मध्य भारतीय हरितोद्भिद-भौगौलिक क्षेत्र के लिए नए हैं।

\section{Abstract}

On the basis of compilation of earlier reports and results of fresh exploration, the present work provides a list of 113 bryophyte taxa from Mount Abu, Rajasthan including 24 taxa as new to the Mount Abu area. Out of these, 14 are new records to Rajasthan state, while 07 taxa are new to Central Indian bryo-geographical region.

Keywords: Central India, Hornworts, Liverworts, Mosses, Rajasthan

\section{INTRODUCTION}

Mount $\mathrm{Abu}$, one of the famous tourist places and only hills station of Rajasthan, is situated between $24^{\circ} 31^{\prime}$ to $72^{\circ} 38^{\prime} \mathrm{N}$ and $72^{\circ} 38^{\prime}$ to $72^{\circ} 53^{\prime} \mathrm{E}$ in the Sirohi district of Southern Rajasthan at an average height of $4500 \mathrm{ft}$. above sea level (Chaudhary \& Deora, 2001) and falls under Central Indian bryo-geographical zone (Alam $\&$ al., 2015). It has been the center of attraction for bryologists and most worked out area of Rajasthan in terms of bryo-diversity. Bapna (1958) published a list of 24 species of Bryophytes from Mount Abu, including 20 liverworts and four hornworts. Later, Bapna \& Vyas (1962) published an updated list of 28 species of liverworts of Mount Abu. Chaudhary \& Deora (1993) reported 42 species of mosses from Rajasthan, including 39 species from Mt Abu. Chaudhary \& Deora (2001) further published Mount Abu specific list of 25 species of mosses under 18 genera. Alam \& al. (2014) compiled an updated list of 46 mosses of Mount Abu, though, without any distributional details. Some of the above mentioned work though attempted to list the species present in the area, however, remain focused on one or other group of bryophytes and none of them provided a consolidated account of all the three major groups (liverworts, hornworts and mosses) altogether. Secondly, the area still harbors a good amount of unexplored diversity as reflected from regular reports of new additions to this area, including present report of about 24 taxa as new to the area. Further, recent taxonomic studies resulting into changes in the taxonomic status of various taxa, underlines the need of an updated account of the bryophytes in the region.

Recently, during an exploration in the Mount Abu region, a total of 29 Bryophyte taxa were observed, including 24 new records to the region. The paper provides a comprehensive and updated list of all the 113 bryophytes taxa in Mount Abu region along with their distributional details in the area.

\section{MATERIALS AND METHODS}

Voucher specimens were collected during March, 2015 along with geo-coordinates and other relevant data and deposited in Bryophyte Herbarium of CSIR-National Botanical Research Institute (LWG). Identification was made with the help of relevant literature and a complete 
list was prepared on the basis of present study as well as earlier reports.

\section{RESULTS}

The present survey and earlier reports altogether revealed the presence of a total of 113 taxa of bryophytes in Mount Abu region, including 28 liverworts, seven hornworts and 78 mosses. This list also includes 24 taxa found for the first time in Mount Abu region. Out of these, 14 taxa are new to Rajasthan bryo-flora while 07 taxa are being reported for the first time from Central Indian bryo-geographical region. The list also provides distributional details of each taxon in Mount Abu region with references of the original reports.

\section{Liverworts (Marchantiophyta)}

Asterella khasiana (Griff.) Pande, K.P. Srivast. \& Sultan Khan, J. Hattori Bot. Lab. 11: 8. 1954.

(Aytoniaceae)

Distribution: Common (Bapna, 1958; Bapna \& Vyas, 1962; both report as A. blumeana Nees).

Asterella wallichiana (Lehm. \& Lindenb.) Pande, K.P. Srivast. \& Sultan Khan ex Grolle, Khumbu Himal. 1(4): 262, 1966. (Aytoniaceae)

Distribution: Very common (Bapna 1958; Bapna \& Vyas, 1962; both report as A. angusta St.)

Calycularia crispula Mitt., J. Proc. Linn. Soc., Bot. 5: 122. 1861. (Allisoniaceae)

Distribution: Hanuman temple, Gaumukh (Bapna, 1958).

However, Singh \& al. (2016) recently mentioned the presence of this species only in Himalayas and South India.

Cyathodium cavernarum Kunze ex Lehm., Nov. Strip. Pug. 6: 18. 1834. (Targioniaceae)

Distribution: Naki lake, Achalgarh (Bapna 1958; Bapna \& Vyas, 1962; both as C. barodae Chavan).

Cyathodium tuberosum Kashyap, New Phytol. 13:210. 1914. (Targioniaceae)

Distribution: Common (Kashyap, 1929, 1932; Bapna, 1958; Bapna \& Vyas, 1962).

Frullania ericoides (Nees ex Mart.) Mont., Ann. Sci. Nat. Bot., Ser. 2, 12: 51. 1839. (Jubulaceae)

Specimens examined: INDIA: Rajasthan, Mount Abu,
Guru Sikhar, N 24³8'333, E 7246'35.5", 1491m, on bark, 18.03.2015, K.K. Rawat 300094A; Achalgarh, N 24'37'05.4", E 7245'52.3", $1306 \mathrm{~m}$, on soil covered rock, 18.03.2015, K.K. Rawat 300095B; on bark, K.K. Rawat 300096B.

Distribution: Guru Sikhar, Achalgarh (present study). New to Rajasthan. In Central India, earlier reported from Madhya Pradesh only (Singh \& al., 2016).

Fossombronia himalayensis Kashyap, New Phytol. 14:4. 1915. (Fossombroniaceae)

Specimen examined: INDIA: Rajasthan, Mount Abu, Guru Sikhar, N 2438'59.5", E 7246’37.8", 1732 m, on soil covered rock, 18.03.2015, K.K. Rawat 300089B.

Distribution: Sunset point (Bapna, 1958), along the path towards Sunset point, Kodra dam (Bapna \& Vyas, 1962), without location details (Srivastava \& Udar, 1975), Guru Sikhar (Present study).

Fossombronia pusilla (L.) Dumort., Recueil. Observ. Jungerm. 11. 1835. (Fossombroniaceae)

Specimen examined: India: Rajasthan, Mount Abu, Guru Sikhar, N 24³8'59.5", E 7246'37.8", 1732 m, on soil, 18.03.2015, K.K. Rawat 300088A, 300090A.

Distribution: Guru Sikhar (present study). New to Central India. Earlier known from Meghalaya, Tamil Nadu, and Uttarakhand (Singh \& al., 2016).

Fossombronia wondraczeckii (Corda) Dumort., Recueil. Observ. Jungerm. 11. 1835. (Fossombroniaceae)

Specimens examined: INDIA: Rajasthan, Mount Abu, Guru Sikhar, N 24³8'59.5", E 7246'37.8", 1732m, on rock, 18.03.2015, K.K. Rawat 300087A, 300091C; Achalgarh, N 24³7'05.4", E 7245'52.3", 1306 m, on soil covered rock, 18.03.2015, K.K. Rawat 300095E.

Distribution: Guru Sikhar, Achalgarh (present study). New to Rajasthan. In Central Indian region, earlier known from Madhya Pradesh (Singh \& al., 2016).

Lejeunea $c f$. aloba Sande Lac. in Dozy, Plagiochila sandei 10. (1856). (Lejeuneaceae)

Specimens examined: India: Rajasthan, Mount Abu, Sun set point, N 24³5'7.3", E 7242'5.2", 1189 m, on bark, 19.03.2015, K.K. Rawat 300105B; near Arbuda Devi Temple, N 24³6'4.9", E 7242'54.7", 1190 m, on Eucalyptus bark, 20.03.2015, K.K. Rawat 300108B.

Distribution: Near Arbuda devi temple, sunset point (present study). New to Rajasthan

Marchantia polymorpha L. Sp. Pl. 1137. 1753 subsp. 
polymorpha (Marchantiaceae)

Distribution: Craig way (Bapna, 1958; Bapna \& Vyas, 1962).

Metzgeria himalayensis Kashyap, J. Bombay Nat. Hist. Soc. 26: 280. 1917. (Metzgeriaceae)

Distribution: Sunset point (Bapna \& Vyas, 1962).

Pellia epiphylla (L.) Corda in Opiz, Naturalientausch 12: 654. 1829. (Pelliaceae)

Distribution: Behind Sirohi Kothi, near Hanuman temple, on way to Gaumukh (Bapna 1958; Bapna \& Vyas, 1962).

Plagiochasma appendiculatum Lehm \& Lindenb. in Lehm., Nov. Strip. Pug. 4: 14. 1832. (Aytoniaceae)

Specimen examined: India: Rajasthan, Mount Abu, On way to Sunset point, N 24³5'7.3", E 7242’5.2", 1189 m, on soil, 19.03.2015, K.K. Rawat 300106A.

Distribution: Very common (Bapna, 1958; Bapna \& Vyas, 1962).

Plagiochasma pterospermum C. Massal., Mem. Accad. Agric. Verona 73: 46. 1897. (Aytoniaceae)

Distribution: Common (Bapna 1958; Bapna \& Vyas, 1962, both report as $P$. articulatum Kash.).

Plagiochasma intermedium Lindenb. \& Gottsche in Gottsche \& al., Syn. Hepat. 513. 1846. (Aytoniaceae)

Specimens examined: India: Rajasthan, Mount $\mathrm{Abu}$, Guru Sikhar, N 24³8'59.5", E 7246’37.8", 1732 m, on soil, 18.03.2015, K.K. Rawat 300085A; same place, on rock, K.K. Rawat 300087B; Gomukh Road, N 24³4'50.8", E 7243'10.3", 1164 m, on soil, 21.03.2015, K.K. Rawat 300112A.

Distribution: Guru Sikhar, Goumukh road (present study); without location details (Srivastava \& al., 1985).

Reboulia hemisphaerica (L.) Raddi, Opusc. Sci. 2(6): 357. 1818. (Aytoniaceae)

Specimen examined: INDIA: Rajasthan, Mount Abu, On way to Sunset point, N 24³5'7.3", E 7242'5.2", 1189 m, on soil, 19.03.2015, K.K. Rawat 300099A, 300106B.

Distribution: Without location details (Bapna \& Kachroo 2000).

Riccia abuensis Bapna, Trans. Brit. Bryol. Soc. 4: 249. 1962. (Ricciaceae)

Distribution: Near a tank towards Sunset point (Bapna, 1962).
Riccia aravalliensis Pande \& Udar, J. Indian Bot. Soc. 36: 249. 1957. (Ricciaceae)

Distribution: Without location details (Pande \& Udar, 1957), Sunset point and Naki lake (Bapna 1958; Bapna \& Vyas, 1962).

Riccia billardieri Mont. et Nees in Gottsche \& al., Syn. Hepat. 602. 1846. (Ricciaceae)

Distribution: Kumarwara, St. Mary High School (Bapna, 1958); common (Bapna \& Vyas, 1962).

Riccia crystallina L., Sp. Pl. 1138. 1753. (Ricciaceae)

Distribution: Oriya, Kodara Dam, Dilwara (Bapna 1958), Sunset point, Anadhra point (Bapna 1958; Bapna \& Vyas, 1962, both reports as R. plana Taylor).

Riccia discolor Lehm. \& Lindenb. in Lehm., Nov. Strip. Pug. 4:1. 1832. (Ricciaceae)

Specimen examined: India: Rajasthan, Mount Abu, Achalgarh, N 243'05.4", E 7245'52.3", 1306 m, on soil, 18.03.2015, K.K. Rawat 300097B.

Distribution: Common (Bapna, 1958, 1965; Bapna \& Vyas, 1962).

Riccia frostii Aust., Bull. Torrey Bot. Club. 6: 17. 1875. (Ricciaceae)

Distribution: Near Sunset point, near old Sirohi Kothi, in a valley near Achalgarh (Bapna, 1965 as 'R. frostrii Aust.'; Bapna \& Vyas, 1962).

Riccia fluitans L., Sp. Pl. 1139. 1753. (Ricciaceae)

Distribution: Near Municipal Park (Bapna, 1958 as ' $R$. fluitanos L.'), on way to sunset point (Bapna \& Vyas, 1962).

Riccia gangetica Ahmad ex L. Söderstr., A. Hagborg \& von Konrat, Phytotaxa 65: 57. 2012. (Ricciaceae)

Distribution: Sunset point, Naki Lake, Achalgarh (Bapna, 1958); common (Bapna \& Vyas, 1962).

Riccia melanospora Kashyap, Liverw. W. Himal. 1: 94.1929. (Ricciaceae)

Distribution: Near Residency garden, Dilwara (Bapna \& Vyas, 1962)

Riccia grollei Udar, Curr. Sci. 34:126. 1965. (Ricciaceae)

Distribution: Kumarwara and Sunset point (Bapna \& Vyas, 1962 as R. tuberculata).

Targionia hypophylla L., Sp. Pl. 1136. 1753. (Targioniaceae) 
Specimens examined: India: Rajasthan, Mount $\mathrm{Abu}$, Guru Sikhar, N 24³8'59.5", E 7246'37.8", 1732 m, on soil covered rock, 18.03.2015, K.K. Rawat 300089A. Achalgarh, N 24³7'05.4", E 7245'52.3", 1306 m, on soil covered rock, 18.03.2015, K.K. Rawat 300095D.

Distribution: Common (Bapna 1958; Bapna \& Vyas, 1962), Guru Sikhar, Achalgarh (present report).

\section{Hornworts (Anthocertophyta)}

Anthoceros erectus Kashyap, New Phytol. 14:9. 1915. (Anthocerotaceae)

Distribution: Behind old Sirohi Kothi, Sunset point (Bapna, 1958; Bapna \& Vyas, 1962).

Anthoceros subtilis Steph., Sp. Hepat. 5: 1003. 1916. (Anthocerotaceae)

Distribution: Sunset point, Dilwara (Bapna \& Vyas, 1962).

Notothylas indica Kashyap, in Kashyap \& N.L. Dutt, Proc. Lahore Phil. Soc. 4: 49. 1925. (Notothyladaceae)

Distribution: Sunset point (Bapna \& Vyas, 1962).

Notothylas levieri Schiffn. ex Steph., Sp. Hepat. 5: 1021. 1917.

Distribution: Near Kumarwara, on way to St Mary School and towards sunset point (Bapna, 1958; Bapna \& Vyas, 1962; Singh \& al., 2016). (Notothyladaceae)

Phaeoceros himalayensis (Kashyap) Prosk. ex Bapna \& C.G. Vyas, J. Hattori Bot. Lab. 25: 88. 1962. (Anthocerotaceae)

Distribution: Towards sunset point, behind old Sirohi Kothi (Bapna \& Vyas, 1962).

Phaeoceros carolinianus (Michx.) Prosk., Bull. Torrey Bot. Clob 78: 347. 1951. (Anthocerotaceae)

Specimen examined: INDIA: Rajasthan, Mount Abu, on way to Sunset point, N 24³5'7.3", E 7242'5.2", 1189 m, on soil, 19.03.2015, K.K. Rawat 300106C.

Distribution: Kodra dam, on way to sunset point (Bapna \& Vyas, 1962 as P. laevis ssp. carolinianus).

Phaeoceros laevis (L.) Prosk., Bull. Torrey Bot. Club 78: 347. 1951. (Anthocerotaceae)

Specimen examined: INDIA: Rajasthan, Mount Abu, Guru Sikhar, N 24³8'59.5", E 7246’37.8", 1732 m, on soil, 18.03.2015, K.K. Rawat 300088C, 300090A, 300091A.
Distribution: Guru Sikhar (present study), Without location details (Asthana \& Srivastava, 1991 as P. laevis subsp. laevis).

\section{Mosses (Bryophyta)}

Anoectangium clarum Mitt., J. Proc. Linn. Bot. Soc. Suppl. 1: 31, 1859. (Pottiaceae)

Distribution: Without location details (Alam \& al., 2014).

Anoectangium stracheyanum Mitt., Musc. Ind. Or. 31, 1859. (Pottiaceae)

Specimen examined: INDIA: Rajasthan, Mount Abu, Guru Sikhar, N 24³8'59.5", E 7246'37.8", 1732 m, on soil, 18.03.2015, K.K. Rawat 300086A.

Distribution: Guru Sikhar (present study); Bharatpur House, Near Nakki Lake, near Hanuman temple, on way to Gaumukh (Bapna \& Chaudhary, 1989; Chaudhary \& Deora, 1993, 2001); without location details (Nath \& Gupta, 2011; Alam \& al., 2014).

Anomobryum auratum (Mitt.) A. Jaeger, Ber. Thätigk. St. Gallischen Naturwiss. Ges. 1873-74: 142. 1875. (Bryaceae)

Specimen examined: INDIA: Rajasthan, Mount Abu, Guru Sikhar, N 24³8'59.5", E 7246’37.8", 1732 m, on soil, 18.03.2015, K.K. Rawat 300088G.

Distribution: Guru Sikhar (present study); Sunset point (Chaudhary \& Deora, 1993, 2001); without location details (Alam \& al., 2014).

Barbula constricta Mitt., J. Proc. Linn. Soc. Bot. Suppl. 1: 33, 1859. (Pottiaceae)

Distribution: Sunset point area, near Nakki lake (Bapna \& Chaudhary, 1989; Chaudhary \& Deora, 1993, 2001); without location details (Alam \& al., 2014).

Barbula indica (Hook.) Spreng., Nomencl. Bot. 2: 72 1824. (Pottiaceae)

Specimens examined: INDIA: Rajasthan, Mount Abu, Achalgarh, N 24³7'05.4", E 7245'52.3", 1306 m, on soil, 18.03.2015, K.K. Rawat 300097D; near Dilwara Temple, N 24³6'27.4", E 7243'22.6", 1153 m, on rock, 20.03.2015, K.K. Rawat 300109B.

Distribution: Achalgarh, Near Dilwara temple (present study). New to Mt Abu region. In Rajasthan, it was earlier known from Udaipur \& Jaipur (Stern, 2000, Alam \& al. 2015). 
Brachymenium acuminatum Harv., Icon. Pl. 1:19. 1836. (Bryaceae)

Distribution: Sunset point area, on way to Trever Tal, near Nakki lake (Chaudhary \& Deora, 1993, 2001); without location details (Alam \& al., 2014).

Brachymenium exile (Dozy \& Molk.) Bosch. \& Sande Lac., Bryol. Jav. 1: 139, 1860. (Bryaceae)

Distribution: Sunset point area, Nakki lake (Chaudhary \& Deora, 1993, 2001), without location details (Alam \& al., 2014).

Brachymenium indicum (Dozy \& Molk.) Bosch \& Sande Lac. Bryol. Jav. 1: 141. 1860. (Bryaceae)

Specimen examined: INDIA: Rajasthan, Mount Abu, Near Dilwara Temple, N 24³6'27.4", E 7243'22.6", 1153 m, on rock, 20.03.2015, K.K. Rawat 300109D.

Distribution: Near Dilwara Temple (present study); without location details (Alam \& al., 2014).

Brachymenium bryoides Hook. ex Schwägr, Sp. Musc. Frond., Suppl. 2: 134. pl. 135. 1824. (Bryaceae)

Specimen examined: INDIA: Rajasthan, Mount Abu, Gomukh Road, N 24³4'50.8", E 7243'10.3", 1164 m, on soil, 21.03.2015, K.K. Rawat 300112B.

Distribution: Goumukh road (present study). New to Rajasthan. In Central Indian region, it was earlier known from Madhya Pradesh (Nath \& Bansal, 2009; Alam \& al., 2015).

Brachymenium himalayanum Dixon, Anniv. Vol. Bot. Gard. Calcutta 182. 1942. (Bryaceae)

Specimens examined: INDIA: Rajasthan, Mount Abu, Guru Sikhar, N 24³8'33", E 7246'35.5", $1491 \mathrm{~m}$, on rock, 18.03.2015, K.K. Rawat 300092A; same place, on bark, K.K. Rawat 300094B; near Arbuda Devi Temple, N 24'36'4.9", E 7242'54.7", 1190 m, on Eucalyptus bark, 20.03.2015, K.K. Rawat 300108A.

Distribution: Guru Sikhar, Near Arbuda Devi temple (present study). New to Central Indian region.

Brachymenium sikkimense Renauld \& Cardot, Bull. Soc. Roy. Bot. Belgique 38 (1): 12. 1900. (Bryaceae)

Specimens examined: INDIA: Rajasthan, Mount Abu, Guru Sikhar, N 24³8'59.5", E 7246'37.8", 1732 m, on soil, 18.03.2015, K.K. Rawat 300088E, 300090B, 300091D; on soil covered rock, K.K. Rawat 300093B.

Distribution: Guru Sikhar (present study). New to Rajasthan. In Central Indian region, it was earlier known from Gujarat (Chaudhary \& al., 2006, Chaudhary \& Sharma, 2007, Alam \& al., 2015).

Brachymenium turgidum Broth. ex Dixon, Rev. Bryol. 35: 94. 1908. (Bryaceae)

Distribution: Guru Sikhar (Chaudhary \& al., 2003).

Bryoerythrophyllum recurvirostrum (Hedw.) P.C. Chen., Hedw. 80: 255. 1941. (Pottiaceae)

Distribution: Anadra point (Bapna \& Chaudhary, 1989); Sunset point area, on way to St. Mary's school (Chaudhary \& Deora, 1993, 2001); without location details (Alam \& al. 2014).

Bryoerythrophyllum recurvum (Griff.) K. Saito, Bull. Univ. Mus. Univ. Tokyo 8: 254. 1975. (Pottiaceae)

Specimen examined: INDIA: Rajasthan, Mount Abu, Guru Sikhar, N 24³8'59.5", E 7246'37.8", 1732 m, on soil, 18.03.2015, K.K. Rawat 300088F.

Distribution: Guru Sikhar (present study). New to Central Indian Region.

Bryum argenteum Hedw., Sp. Musc. Frond., 181, 1801. (Bryaceae)

Specimen examined: INDIA: Rajasthan, Mount Abu, Guru Sikhar, N 2438'59.5", E 7246'37.8", 1732 m, on soil, 18.03.2015, K.K. Rawat 300086B.

Distribution: Guru Sikhar (present study); Sunset point area (Chaudhary \& Deora, 1993); on way to Traver Tal, Sunset point area (Deora \& Chaudhary, 1996; Bansal \& Nath 2014); without location details (Alam \& al., 2014).

Bryum cellulare Hook., Sp. Musc. Frond. Suppl. 3(1): 214, 1827. (Bryaceae)

Distribution: Sunset Point, Achalgarh (Chaudhary \& Deora, 1993; Deora \& chaudhary, 1996); near Dilwara temples (Bansal \& Nath, 2014).

Bryum dunense A.J.E. Sm. \& H. Whitehouse, J. Bryol. 10: 41. 1978. (Bryaceae)

Distribution: Near Dilwara temples (Bansal \& al., 2012; Bansal \& Nath, 2014).

Bryum mildeanum Jur. Verh. K.K. Zool.-Bot. Ges. Wien 12: 967. 1862. (Bryaceae)

Distribution: Sunset point area, Trever Tal, near Dilwara temple (Ellis \& al., 2013 as Bryum apiculatum Schwägr.).

Bryum paradoxum Schwägr., Sp. Musc. Frond., Suppl. 3(1): 224. 1827. (Bryaceae) 
Distribution: Near Nakki lake, on way to Trevor tal (Chaudhary \& Deora, 1993; Deora \& Chaudhary, 1996; Bansal \& Nath, 2014); without location details (Alam \& al., 2014).

Bryum pseudotriquetrum var. subrotundum (Brid.) Gangulee, Mosses E. India 4: 994, 1974. (Bryaceae)

Distribution: Near Dilwara temples (Bansal \& Nath, 2014).

Bryum recurvulum Mitt., J. Proc. Linn. Soc., Bot., Suppl. 1: 74. 1859. (Bryaceae)

Distribution: Near Nakki lake, Trever Tal area (Chaudhary \& Deora, 1993; Deora \& Chaudhary, 1996; Bansal \& Nath, 2014), without location details (Alam \& al., 2014).

Bryum thomsonii Mitt., J. Proc. Linn. Soc., Bot. suppl. 1: 73. 1859. (Bryaceae)

Distribution: Near Dilwara temples (Ellis \& al., 2013, Bansal \& Nath, 2014); without location details (Alam \& al., 2014).

Bryum uliginosum (Brid) Bruch. \& Schump., Bryol. Eur.4:88. 1839. (Bryaceae)

Specimen examined: INDIA: Rajasthan, Mount $\mathrm{Abu}$, near Sunset point, N 24³5'7.3", E 7242'5.2", 1189 m, on bark, 19.03.2015, K.K. Rawat 300105C.

Distribution: Near Sunset point (present study); without location details (Bansal \& Nath, 2014, Alam \& al., 2014).

Diaphanodon procumbens (Müll.) Renauld \& Cardot, Bull. Soc. Roy. Bot. Belgique 38(1): 24.1900. (Trachypodaceae)

Distribution: Sunset point area (Chaudhary \& Deora, 1993); without location details (Alam \& al., 2014).

Didymodon vinealis (Brid.)R. H. Zander, Phytologia 41:25. 1978. (Pottiaceae)

Distribution: Sunset point area, Traver tank area (Bapna \& Chaudhary, 1989; Chaudhary \& Deora, 1993, 2001, all reports as Barbula vinealis Brid.).

Entodon concinnus (De Not.) Paris, Index Bryol. (ed. 2) 2: 130. 1904. (Entodontaceae)

Distribution: Without location details (Alam \& al., 2014).

Entodon myurus (Hook.) Hampe, Linnaea 20: 82. 1847. (Entodontaceae)
Distribution: Near Nakki lake (Chaudhary \& Deora, 1993); without location details (Alam \& al., 2014).

Entodon plicatus Müll. Hal., Linnaea 18: 706. 1845. (Entodontaceae)

Distribution: Without location details (Alam \& al., 2014).

Entodon prorepens (Mitt.) A. Jaeger, Ber. Thätigk. St. Gallischen Naturwiss. Ges. 1876-77: 294. 1878. (Entodontaceae)

Distribution: Sunset point area, near Nakki lake (Chaudhary \& Deora, 1993, 2001).

Entodontopsis tavoyensis (Hook. ex Harv.) W.R. Buck \& R.R. Ireland, Nova Hedwigia 41: 105. 1985. (Entodontaceae)

Specimen examined: INDIA: Rajasthan, Mount Abu, on way to Sunset point, N 24³5'7.3", E 7242'5.2", 1189 m, on bark, 19.03.2015, K.K. Rawat 300098A.

Distribution: on way to Sunset point (present study). New to Central Indian Region.

Entosthodon wallichii Mitt., J. Proc. Linn. Soc., Bot., suppl. 1: 55. 1859. (Funariaceae)

Distribution: Without location details (Nath \& al., 2009).

Erpodium mangiferae Müll. Hal., Linnaea 37: 178, 1872. (Erpodiaceae)

Specimen examined: INDIA: Rajasthan, Mount Abu, Achalgarh, N 24³7'05.4", E 7245'52.3", 1306 m, on bark, 18.03.2015, K.K. Rawat 300096D.

Distribution: Achalgarh (present study). New to Rajasthan. In Central Indian region, it was earlier reported from Gujarat, Jharkhand \& Madhya Pradesh (Chaudhary \& al., 2006; Chaudhary \& Sharma 2007; Gangulee 1974-78; Singh \& Kaul 2002; Handoo \& al., 2009; Nath \& al., 2007; Alam \& al., 2015).

Fabronia minuta Mitt., J. Proc. Linn. Soc., Bot. suppl. 1: 76. 1859. (Fabroniaceae)

Distribution: Sunset point area, near Sirohi Kothi (Chaudhary \& Deora, 1993); without locality (Alam \& al. 2014).

Fissidens bryoides Hedw. Sp. Musc., Frond. 153. 1801. (Fissidentaceae)

Specimens examined: INDIA: Rajasthan, Mount Abu, Guru Sikhar, N 24³8'59.5", E 7246’37.8", 1732 m, on 
soil, 18.03.2015, K.K. Rawat 300088D; same place, on soil covered rock, 300089C; on way to Sunset point, N 24³5'7.3", E 7242'5.2", 1189 m, on soil, 19.03.2015, K.K. Rawat 300099B; Tiger path, N 24\%34'14.3", E 72'43'28.1", 1176 m, on bark, 21.03.2015, K.K. Rawat $300111 \mathrm{~A}$.

Distribution: Guru Sikhar, Tiger path, on way to Sunset point (present study); Sunset point road (Chaudhary \& Deora, 1993).

Fissidens crispulus Brid., Musc. Rec. suppl. 4: 187. 1819. (Fissidentaceae)

Distribution: On way to St. Mary's School (Chaudhary \& Deora, 1993, 2001 as F. sylvaticus Griff.).

Fissidens diversifolius Mitt., J. Proc. Linn. Soc., Bot., Suppl. 1: 140. 1859. (Fissidentaceae)

Distribution: Ada Lakda Municipal post, on way to St Mary's School, Kumarwara (Bapna, 1980); on way to St Mary's School, on way to sunset point, near Kamla Nehru Park (Chaudhary \& Deora, 1993, 2001), without locality (Alam \& al., 2014).

Fissidens geminiflorus Dozy \& Molk., Pl. Jungh. 316.1854. (Fissidentaceae)

Distribution: Kodara Dam, Sirohi-Mandar about 36 miles from Sirohi (Bapna, 1980 as F. geminiflorus var. nagasakinus Iwats.); near Dilwara temple, near Nakki lake (Chaudhary \& Deora, 1993, 2001 as F. geminiflorus var. nagasakinus (Besch.) Z. Iwats.), without location details (Alam \& al. 2014).

Fissidens involutus ssp. curvato-involutus (Dixon.) Gangulee, Mosses E India 2: 548. 1971. (Fissidentaceae)

Distribution: Near Municipal Park (Bapna, 1980); on way to sunset point, near Nakki lake, near Municipal Park (Chaudhary \& Deora, 1993, 2001) and without location details (Alam \& al., 2014), all reports as $F$. curvato-involutus Dix.

Fissidens minutus Thwaites \& Mitt., J. Linn. Soc., Bot.13: 323. 1873. (Fissidentaceae)

Specimen examined: India: Rajasthan, Mount Abu, Achalgarh, N 24³7'05.4", E 7245'52.3", 1306 m, on bark, 18.03.2015, K.K. Rawat 300096A.

Distribution: Achalgarh (present study). New to Central Indian region.

Fissidens orishae Gangulee Nova Hedwigia 8: 140. 1964. (Fissidentaceae)
Specimen examined: India: Rajasthan, Mount Abu, on way to Sunset point, N 24³5'7.3", E 7242'5.2", 1189 m, on bark, 19.03.2015, K.K. Rawat 300100C.

Distribution: On way to Sunset point (present study). New to Rajasthan. In Central Indian region, earlier known from Madhya Pradesh (Kapoor \& al., 2011; Alam \& al., 2015).

Fissidens subpalmatus Müll. Hal., Linnaea 37: 164. 1872. (Fissidentaceae)

Specimen examined: India: Rajasthan, Mount Abu, Guru Sikhar, N 24³8'59.5", E 7246'37.8", 1732 m, on rock, 18.03.2015, K.K. Rawat 300087C; same place, on soil covered rock, 300095B.

Distribution: Guru Sikhar (present study). New to Rajasthan. The species was earlier known from Central India without details of location (see Lal, 2005; Alam $\&$ al., 2015), hence present report confirms its presence in the region.

Fissidens sylvaticus var. auriculatus (Müll. Hal.) Gangulee, Mosses E India 2: 540. 1971. (Fissidentaceae)

Distribution: Without location details (Alam \& al., 2014).

Fissidens sylvaticus var. teraicola (Müll. Hal.) Gangulee, Mosses E India 2: 539. 1971. (Fissidentaceae)

Distribution: Without location details (Alam \& al., 2014).

Fissidens taxifolius Hedw., Sp. Musc. Frond. 155. pl. 39: f. 1-5. 1801. (Fissidentaceae)

Specimen examined: INDIA: Rajasthan, Mount Abu, on way to Sunset point, N 24³5'7.3", E 7242'5.2", 1189 m, on soil, 19.03.2015, K.K. Rawat 300106D.

Distribution: On way to Sunset point (present study). New to Rajasthan.

Funaria hygrometrica Hedw., Spec. Musc. Frond. 172. 1801. (Funariaceae)

Distribution: Sunset point area, Nakki lake (Chaudhary \& Deora, 1993, 2001); without location details (Alam \& al., 2014).

Gemmabryum apiculatum (Schwägr.) J.R. Spence \& H.P. Ramsay, Phytologia 87: 65. 2005. (Bryaceae)

Distribution: Trevor Tal, Sunset point (Chaudhary \& Deora, 1993 as Bryum plumosum Dozy. ex Molk.); without location details (Alam \& al., 2014).

Gemmabryum exile (Dozy. \& Molk.) J.R. Spence \& 
HP Ramsey, Phytologia 87: 67. 2005. (Bryaceae)

Specimen examined: India: Rajasthan, Mount $\mathrm{Abu}$, Achalgarh, N 24³7'05.4", E 7245'52.3", 1306 m, on soil, 18.03.2015, K.K. Rawat 300097A.

Distribution: Achalgarh (Present study). New to $\mathrm{Mt}$ Abu.

Gymnostomiella vernicosa (Hook. ex Harv.) M. Fleisch., Musci Buitenzorg, 1: 310. 1904. (Pottiaceae)

Specimen examined: INDIA: Rajasthan, Mount Abu, Near Dilwara Temple, N 2436'27.4", E 7243'22.6", 1153 m, on rock, 20.03.2015, K.K. Rawat 300109A.

Distribution: Very common, near Dilwara Temple (present study); Nakki lake, Hanuman temple, Kamla Nehru Park, Sunset point area, Sirohi Kothi (Chaudhary \& Deora, 1993; Chaudhary \& Deora, 2001); without location details (Alam \& al., 2014).

Gymnostomum calcareum Nees \& Hornsch., Bryo. Germ. 1: 153. 1823. (Pottiaceae)

Distribution: Without location details (Bapna \& Chaudhary; 1989).

Hydrogonium arcuatum (Griff.) Wijk. \& Margad, Taxon 7: 289. 1958. (Pottiaceae)

Distribution: Near Sirohi Kothi, near Nakki lake (Bapna \& Chaudhary, 1989; Chaudhary \& Deora, 1993, 2001); without location details (Alam \& al., 2014).

Hydrogonium consanguineum (Thwaites \& Mitt.) Hilp. Beih., Bot. Centralbl. Abt. 2 50(2): 626. 1933. (Pottiaceae)

Distribution: Sunset point area (Bapna \& Chaudhary, 1989; Chaudhary \& Deora, 1993, 2001); without location details (Alam \& al., 2014).

Hymenostylium recurvirostrum (Hedw.) Dixon, Rev. Bryol. Lichenol. 6: 96. 1933. (Pottiaceae)

Distribution: Bharatpur House (Bapna \& Chaudhary, 1989).

Hyophila involuta (Hook.) A. Jaeger, Ber. S. Gall. Naturew. Ges. 1871-72: 356. 1873. (Pottiaceae)

Specimens examined: INDIA: Rajasthan, Mount Abu, Guru Sikhar, N 24³8'33”, E 7246'35.5", 1491 m, on soil covered rock, 18.03.2015, K.K. Rawat 300093A; on way to Sunset point, N 24³5'7.3", E 7242'5.2", 1189 $\mathrm{m}$, on rock, 19.03.2015, K.K. Rawat 300101A; near Dilwara temple, N 24³6'27.4", E 7243'22.6", 1153 m, on rock, 20.03.2015, K.K. Rawat 300109C.
Distribution: Guru Sikhar, on way to Sunset point, near Dilwara temple (present study); Municipal Post, Achalgarh, Nakki Lake, (Bapna \& Choudhary, 1989 as Gymnostomum involutum Hook.); Sunset point, near Dilwara temple (Chaudhary \& Deora, 1993, 2001), without location details (Alam \& al., 2014).

Hyophila nymaniana (M. Fleisch.) M. Menzel. Willdenowia 22: 198. 1992. (Pottiaceae)

Specimen examined: INDIA: Rajasthan, Mount Abu, Achalgarh, N 2437'05.4", E 7245'52.3", $1306 \mathrm{~m}$, on soil covered rock, 18.03.2015, K.K. Rawat 300095E; on bark, K.K. Rawat 300096C; on way to Sunset point, N 24'35'7.3", E 7242'5.2", 1189 m, on bark, 19.03.2015, K.K. Rawat 300100A, 300103A, 300104A; Tiger path, N 24³4'14.3", E 7243'28.1", $1176 \mathrm{~m}$, on soil, 21.03.2015, K.K. Rawat 300110B.

Distribution: Achalgarh, on way to Sunset point, Tiger path (present study). New to Rajasthan. In Central Indian region, earlier known from Chhattisgarh, Gujarat and Madhya Pradesh (Nath \& Gupta, 2011; Aziz \& Vohra, 2008; Nath \& al., 2007; Gupta \& al., 2013; Alam \& al., 2015).

Hyophila spathulata (Harv.) A. Jaeger, Ber. Thätigk. St. Gallischen Naturwiss. Ges. 1871-72: 353, 1873. (Pottiaceae)

Distribution: Without location details (Alam \& al., 2014). (Fabroniaceae)

Levierella neckeroides (Griff.) O'Shea \& Matcham, J. Bryol. 27: 98. 2005.

Distribution: Near Kamla Nehru Park (Chaudhary \& Deora, 1993 as Levierella fabroniacea Müll. Hal.); without location details (Alam \& al., 2014).

Loiseaubryum nutans (Mitt.) Fife, J. Hattori Bot. Lab. 58: 192. 1985. (Funariaceae)

Distribution: Sunset point area (Chaudhary \& Deora 1993 as Funaria nutans (Mitt.) Broth.); without location details (Alam \& al., 2014).

Philonotis mollis (Dozy \& Molk.) Mitt., J. Proc. Linn. Soc., Bot. Suppl. 1: 60. 1859. (Bartramiaceae)

Distribution: Sunset point area, near Nakki lake (Chaudhary \& Deora, 1993, 2001); without location details (Alam \& al., 2014).

Philonotis leptocarpa Mitt. J. Proc. Linn. Soc., Bot., Suppl. 1: 60. 1859. (Bartramiaceae)

Specimen examined: INDIA: Rajasthan, Mount Abu, 
Tiger Path, N 24³4'14.3", E 7243'28.1", 1176 m, on soil, 21.03.2015, K.K. Rawat 300110D.

Distribution: Tiger Path (present study). New to Central Indian region.

Philonotis thwaitesii Mitt., J. Proc. Linn. Soc., Bot. Suppl. 1: 60. 1859. (Bartramiaceae)

Distribution: Sunset point area, near Nakki lake (Chaudhary \& Deora, 1993 as Philonotis revoluta Bosch \& Sande Lac.); without location details (Alam \& al., 2014).

Physcomitrium japonicum (Hedw.) Mitt., Trans. Linn. Soc., London, Bot. 3: 164, 1891. (Funariaceae)

Distribution: Near a tank on way to sunset point (Chaudhary \& Deora, 1993); near sunset point (Chaudhary \& Deora, 2001).

Plagiothecium cavifolium (Brid.) Z. Iwats., J. Hattori Bot. Lab. 33: 360. 1970. (Plagiotheciaceae)

Distribution: Without location details (Alam \& al., 2014).

Pleurochaete squarrosa (Brid.) Lindb., Öfvers. Förh. Kongl. Svenska Vetenzk.-Akad. 2: 253. 1864. (Pottiaceae)

Distribution: Kodra Dam (Bapna \& Chaudhary, 1989).

Pseudobarbella compressiramea (Renauld \& Cardot) Nog. in. Hara, Fl. E. Himalaya 574. 1966. (Meteoriaceae)

Distribution: Sunset point area, Nakki lake (Chaudhary \& Deora, 1993); without location details (Alam \& al., 2014).

Ptychostomum capillare (Hedw.) D.T. Holyoak \& N. Pederson, J. Bryol. 29: 119. 2007. (Bryaceae)

Specimen examined: India: Rajasthan, Mount Abu, Tiger Path, N 24³4'14.3", E 7243'28.1", 1176 m, on soil, 21.03.2015, K.K. Rawat 300110C.

Distribution: Tiger Path (present study); Sunset point area, near Nakki lake (Chaudhary \& Deora, 1993; Deora \& Chaudhary, 1996, both reports as Bryum capillare L. ex Hedw.); near Dilwara temple, Guru Sikhar, Shri Ragunath Rai inn (Bansal \& Nath, 2014, as Bryum capillare); without location details (Alam \& al., 2014).

Semibarbula orientalis (F. Weber) Wijk. \& Margad., Taxon 8: 75, 1959. (Pottiaceae)

Distribution: Nakki Lake, Achalgarh (Bapna \& Chaudhary, 1989); Sunset point area, Nakki lake
(Chaudhary \& Deora, 1993, 2001); without location details (Alam \& al., 2014).

Stereophyllum decorum (Mitt.) Wijk. \& Marg. Taxon 9: 52. 1960. (Stereophyllaceae)

Specimen examined: India: Rajasthan, Mount Abu, near sunset point, N 24'35'7.3", E 72'42'5.2", $1189 \mathrm{~m}$, on bark, 19.03.2015, K.K. Rawat 300104C.

Distribution: Near Sunset point (present study). New to Rajasthan. In Central Indian region, the species was earlier known from Gujarat and Madhya Pradesh (Singh \& Kaul, 2002; Chaudhary \& al., 2006; Chaudhary \& Sharma, 2007).

Stereophyllum tavoyense (Hook. ex Harv.) A. Jaeger, Ber. Thätigk. St. Gallischen Naturwiss. Ges. 1877-78: 279. 1880. (Stereophyllaceae)

Distribution: Without location details (Alam \& al., 2014).

Stereophyllum wightii (Mitt.) A. Jaeger., Ber. Thätigk. St. Gallischen Naturwiss. Ges. 1877-78: 279. 1880. (Stereopjyllaceae)

Specimen examined: INDIA: Rajasthan, Mount Abu: on way to Sunset point, N 24³5'7.3", E 7242'5.2", 1189 $\mathrm{m}$, on bark, 19.03.2015, K.K. Rawat 300103C, 300105A.

Distribution: On way to Sunset point (present study). New to Rajasthan. In Central Indian region, it was earlier reported from Jharkhand and Madhya Pradesh (Gangulee, 1978-80; Singh \& Kaul, 2002; Handoo \& al., 2009; Alam \& al., 2015).

Timmiella anomala (Bruch \& Schimp.) Limpr., Laubm. Deutschl. 1: 592. 1888. (Pottiaceae)

Distribution: Trevor Tal, on way to Parasram Ji, Sunset point, Travor tank (Chaudhary \& Deora, 1993, 2001); without location details (Alam \& al., 2014)

Timmiella diminuta (Müll. Hal.) P.C. Chen, Hedwigia 80: 176.1941. (Pottiaceae)

Specimen examined: INDIA: Rajasthan, Mount Abu, on way to Sunset point, N 24³5'7.3", E 7242'5.2", 1189 m, on soil, 19.03.2015, K.K. Rawat 300106E.

Distribution: Sunset point (present study); without location details (Bapna \& Chaudhary, 1989).

Tortula muralis Hedw., Sp. Musc. Frond. 123. 1801. (Pottiaceae)

Distribution: Near Gaumukh (Chaudhary \& Deora, 1993, 2001); without location details (Alam \& al., 2014; 
Alam \& al., 2015).

Trematodon subulosus Griff. Calcutta J. Nat. Hist. 2: 493. 1842. (Bruchiaceae)

Distribution: Sunset point, Nakki Lake (Bapna \& Choudhary, 1989; Chaudhary \& Deora, 1993, 2001); without location details (Alam \& al., 2014).

Trichostomum tenuirostre (Hook. \& Taylor) Lindb., Öfvers. Förh. Kongl. Svenska Vetensk.-Akad. 21(4): 225. 1864. (Pottiaceae)

Specimen examined: INDIA: Rajasthan, Mount Abu, Guru Sikhar, N 24³8'33", E 7246'35.5", 1491 m, on rock, 18.03.2015, K.K. Rawat 300092B.

Distribution: Guru Sikhar (present study). New to Central Indian region.

Weissia controversa Hedw., Sp. Musc. Frond. 67.1801. (Pottiaceae)

Distribution: Near Bharatpur House (Bapna \& Chaudhary, 1989), Nakki lake, Sunset point area (Chaudhary \& Deora, 1993, 2001); without location details (Alam \& al., 2014).

Weissia ghatensis Dixon \& P. de la Varde, Ann. Cryptog. Exot. 3: 174. 1930. (Pottiaceae)

Specimen examined: INDIA: Rajasthan, Mount Abu, Guru Sikhar, N 24³8'59.5", E 7246'37.8", 1732 m, on soil, 18.03.2015, K.K. Rawat 300088B.

Distribution: Guru Sikhar (present study). New to Rajasthan. In Central Indian region, it was earlier reported without any location details (Lal, 2005; Alam \& al., 2015). The report confirms its presence in the region.

Weissia edentula Mitt., J. Proc. Linn. Soc., Bot., Suppl. 1: 27. 1859. (Pottiaceae)

Specimen examined: INDIA: Rajasthan, Mount Abu, Achalgarh, N 24³7'05.4", E 7245'52.3", 1306 m, on soil covered rock, 18.03.2015, K.K. Rawat 300095A.

Distribution: Achalgarh (present study). New to Rajasthan. In Central India, it was earlier reported from Madhya Pradesh (Nath \& al., 2007; Nath \& Gupta, 2011; Alam \& al., 2015).

Wijkia tanytricha (Mont.) H.A. Crum, Bryologist 74: 174. 1971. (Sematophyllaceae)

Distribution: Near Sirohi Kothi, near Nakki lake (Chaudhary \& Deora, 1993; without location details (Alam \& al., 2014).

\section{ACKNOWLEDGEMENTS}

Authors are thankful to the Director, CSIR-NBRI for providing the necessary facilities. We are also thankful to Forest department, Mount Abu for permitting the study work. KKR is thankful to Dr. DK Upreti, Ex-HoD, Plant Diversity, Systematics \& Herbarium Division, CSIR-NBRI, for providing the opportunity to be the part of the survey team. Thanks are also due to Drs AK Asthana and Rajesh Bajpai from CSIR-NBRI, for their help and support during the study. This manuscript bears institutional manuscript number 'CSIRNBRI_MS/2021/01/01'.

\section{REFERENCES}

ASTHANA A. K. AND S. C. SRIVASTAVA 1991. Indian Hornworts (A taxonomic study). Bryophytorum Bibliotheca Band 42: 1-158.

ALAM A., S. PANDEY, V. SINGH, S.C. SHARMA AND V. SHARMA 2014. Moss flora of Mount Abu (Rajasthan), India: An updated checklist. Tropical Plant Research1(1): 8-13.

ALAM A., K.K. RAWAT, P.K. VERMA, V. SHARMA AND D.S. GUPTA 2015. Moss flora of Central India. Plant Science Today 2(4): 159-171.http://dx.doi.org/10.14719/ pst.2015.2.4.126.

AZIZ N.M. AND J.N. VOHRA 2008. Pottiaceae (Musci) in India. Bishen Singh Mahendra Pal Singh, Dehradun, India, $366 \mathrm{pp}$.

BANSAL P. AND V.NATH 2014. Present status of genus Bryum Hedw. (Bryophyta) in Rajasthan, India. GJournal of Environmental Science \& Technology 1(6): 118-122. http://www.gjestenv.com.

BANSAL P., V. NATH AND N.PANDE 2012. Bryum dunense Smith et Whitehouse (Bryophyta: Bryaceae): New to Central India. Proc. Natl. Acad. Sci. India, Sect. B. Biol. Sci.82(3): 453-454.

BAPNA K.R. 1958. A note on hepatic flora of Mt. Abu. Curr. Sci. 7: 259-260.

BAPNA K.R. 1962. A new species of Riccia from Mount Abu (India). Trans. British Bryol. Soc. 4: 249-253.

BAPNA K.R. 1965. A note on the hepatics of Udaipur. Sci. \& Cult. 32: 526.

BAPNA K. R. 1980. Occurrence of Fissidens Hedw. in Rajasthan. J. Indian Bot. Soc. 59: 20-26.

BAPNA K.R. AND B.L. CHAUDHARY 1989. Mosses of Rajasthan-II. Dicranales \& Pottiales. J. Indian Bot. Soc. 68:379-388. 
BAPNA K.R. AND P. KACHROO 2000. Hepaticology in India - II. Himanshu Publication, Delhi, pp. 490.

BAPNA K.R. AND G.G. VYAS 1962. Studies in the liverworts of Mount Abu (India). I. A preliminary account. J. Hattori Bot. Lab. 25: 81-90.

CHAUDHARY B.L., T.P. SHARMA ANDC.SANDAYA 2006. Bryophyte flora of Gujarat (India). Himanshu Publications, Udaipur, New Delhi, India, pp. 287-330.

CHAUDHARY B.L. AND G.S. DEORA 1993. Moss flora of Rajasthan (India). Himanshu Publication, Delhi, India. $127 \mathrm{pp}$.

CHAUDHARY B.L. AND G.S. DEORA 2001. The mosses of Mt. Abu (India) -I. In:Perspective in Indian Bryology, (eds.V.NATH \&A.K.ASTHANA), Bishen Singh Mahendra Pal Singh, Dehradun, pp. 87-125.

CHAUDHARY B.L. AND T.P. SHARMA 2007. Epiphytic bryophytes of Gujarat I, India. In: Current Trends in Bryology, (eds NATH, V. \& ASTHANA, A.K.) Bishen Singh Mahendra Pal Singh, Dehradun, India. Pp. 287330 .

CHAUDHARY B.L., T.P. SHARMA AND C. SANADHYA 2003. Brachymenium turgidum Broth. ex Dix. A new record from Mt. Abu, Rajasthan, India. Phytomorphology 53: 257-259.

DEORA G.S. AND B.L. CHAUDHARY 1996. Occurrence of 2145 .

ELLIS, L.T., S.C. ARANDA, A. K. ASTHANA, P. BANSAL, V. NATH, V. SAHU, J. BAYLISS, G. ASTHANA, S. SRIVASTAVA, S. YADAV, S.M. BRUGUE, M. J. CANO, M.V. DULIN, V. FUDALI, E. FUERTES, R. GABRIEL, F. PEREIRA, V. SILVA, S.R. GRADSTEIN, P. HA JKOVA, V. HA JEK, P. HERAS, M. INFANTE, M. LEBOUVIER, J. MARKA, K.K. NEWSHAM, R. OCHYRA, J. PANTOVIC', M.S. SABOVLJEVIC', N. PHEPHU, J. VAN ROOY, D.A.PHILIPPOV, R.D. PORLEY, F. PUCHE, A. SCHÄ FER-VERWIM, J.G. SEGARRA-MORAGUES, C. SE'RGIO, V.R. SMITH, S.S. TEFANUT, A.J. VANA, AND M.J. WIGGINTON 2013. New National \& Regional Bryophyte Records 37.J. Bryol. 35(4): 290305.DOI: 10.1179/1743282013Y.0000000073.

GUPTA R., V. NATH AND A.K. ASTHANA 2013. Present scenario of moss diversity at Tamia hills \& Patalkot valley (Madhya Pradesh) India. Natl. Acad. Sci. Lett. 36(6): 629-634.https://doi.org/10.1007/s40009-0130182-7.

HANDOO O.N., G.T. DABHATE, S. RAI AND P.K. RAI 2009. Mosses of Pachmarhi.Indian J. Appl. Pure Biol. 24(2): 505-520.
KAPOOR R., V. NATH AND A.K. ASTHANA 2011. Bryophytes of Kanha National Park (Madhya Pradesh), India. Nelumbo 53: 140-144.

KASHYAP S.R. 1929. Liverworts of the Western Himalayas \& the Punjab Plain I. The University of the Panjab, Lahore. pp. 129.

KASHYAP S.R. 1932. Liverworts of the Western Himalayas \& the Punjab Plain II. The University of the Panjab, Lahore. pp. 137.

LAL J. 2005. A checklist of Indian mosses. Bishen Singh Mahendra Pal Singh, Dehradun.

NATH V., V. AWASTHI AND A.K.ASTHANA 2009. Entosthodon wallichii Mitt.- a new addition to the moss flora of Mount Abu (Rajasthan), India. Annal. Forestry 17(2):329-331.

NATH V. AND P. BANSAL 2009. Bryophyte diversity of Bhimbetka world heritage site, Madhya Pradesh (India). J. Indian Bot. Soc. 88(3\&4): 129-140.

NATH V. AND R. GUPTA 2011. An overview of family Pottiaceae (Bryopsida) in Central India with special reference to Pachmarhi Biosphere Reserve (PBR). Lindbergia 34:30-39.

NATH V., A.K. ASTHANA AND R.KAPOOR 2007. Enumeration of the mosses in Amarkantak (Madhya Pradesh), India-I. Taiwania 52(2): 168-176. DOI: 10.6165/tai.2007.52(2).168.

PANDE S.K. AND R. UDAR 1957. A species of Riccia, $R$. aravalliensis Pande \& Udar sp. nov., from Mt. Abu, Rajasthan, India. J. Indian Bot. Soc. 36: 564-579.

SINGH D.K., S.K. SINGH AND D. SINGH 2016. Liverworts and Hornworts of India. An annotated checklist. Botanical Survey of India, Kolkata.

SINGH V.P. AND A. KAUL 2002. Biodiversity and vegetation of Pachmarhi hills. Scientific Publishers, New Delhi, India. i-vii+353 pp.

SRIVASTAVA S.C. AND R. UDAR 1975. The genus Fossombronia Raddi in India, with a note on the Indian taxa of the family Fossombroniaceae. Nova Hedwigia. 26: 799-845.

SRIVASTAVA S.C., D. KUMAR AND A. AGARWAL 1985. The status of Plagiochasma intermedium L. et G. in India. Proc. Indian Acad. Sci., Pl. Sci. 95:365-371.

STERN R.C. 2000. Some bryophytes collected in Rajasthan. J. Bryol. 22(4): 298-299. https://doi.org/10.1179/ jbr.2000.22.4.298. 